\title{
Los determinantes de confianza y riesgo percibido sobre los usuarios de bitcoin
}

\section{The determinants of trust and perceived risk on bitcoin users}

Mg. Carlos Roberto López-Zambrano es estudiante del Doctorado en Desarrollo Regional en el Centro de Investigación en Alimentación y Desarrollo A.C. (México) (carlos.lopezdr18@estudiantes.ciad.mx) (https://orcid.org/0000-00027880-7387)

Dr. Mario Camberos-Castro es profesor emérito del Centro de Investigación en Alimentación y Desarrollo A.C. (México) (mcamberos@ciad.mx) (https://orcid.org/0000-0002-3271-2980)

Dra. Edna María Villarreal-Peralta es docente e investigadora de la Universidad de Sonora (México) (edna.villarreal@unison.mx) (https://orcid.org/0000-0003-3676-3563)

\section{Resumen}

Uno de los posibles determinantes de la intención de usar bitcoin puede ser la confianza de los usuarios, ya que en el poco tiempo de vigencia de la criptomoneda ha demostrado ser una opción real frente al dinero fiduciario. En este aspecto, cabe añadir que existen pocos estudios que consideran a la confianza como un determinante del uso de bitcoin, por lo que el objetivo de este estudio es investigar los factores en los que se basa la confianza y conocer hasta qué punto el riesgo percibido tiene una connotación negativa sobre el uso de la criptomoneda. Para ello se integra un modelo que es analizado bajo la metodología de ecuaciones estructurales por mínimos cuadrados parciales (PLS-SEM), aplicado a una muestra de 174 usuarios de bitcoin. Los resultados de la evaluación de siete hipótesis teóricas indican que los elementos clave de la confianza son las garantías estructurales y la familiaridad ya que determinan la intención de uso y este a su vez el uso real; a diferencia de la confianza basada en las garantías estructurales y la normalidad situacional que son poco significativas. El riesgo percibido demostró tener poca relación con la intención de uso. Por lo tanto, los proveedores de servicios relacionados con bitcoin deben enfocarse en generar situaciones de confianza para los usuarios basadas en la seguridad y las regulaciones, además de crear entornos que generen familiaridad.

\begin{abstract}
One of the possible determinants of the intention to use bitcoin may be the trust of users because in the short time of validity of the cryptocurrency has proven to be a real option against fiat money. In this regard it should be added that few studies consider trust as a determinant of the use of Bitcoin; therefore, the purpose of this research is to know what are the factors on which trust is based and to know to what extent the perceived risk has a negative connotation on the use of cryptocurrency. To accomplish this, a model is integrated and is analyzed under the methodology of structural equations by partial least squares (PLS-SEM), applied to a sample of 174 bitcoin users. The evaluation of the seven theoretical hypotheses indicates that the key elements of trust are structural guarantees and familiarity since they determine the intention of use and this, in turn, the actual use; unlike calculative-based trust and situational normality, which are not very significant. The perceived risk was shown to have little relation to the intended use. Therefore, Bitcoin-related service providers should focus on generating trust situations for users based on security and regulations and creating environments that generate familiarity.
\end{abstract}

\section{Palabras clave I keywords}

Confianza, riesgo percibido, intención de uso, bitcoin, transacciones digitales, modelo PLS-SEM, garantías estructurales. Trust, perceived risk, behavioral intention, bitcoin, digital transactions, PLS-SEM model, structural assurances.

Cómo citar: López-Zambrano, C. R., Camberos-Castro, M., y Villarreal-Peralta, E. M. (2021). Los determinantes de confianza y riesgo percibido sobre los usuarios de bitcoin. Retos Revista de Ciencias de la Administración y Economía, 11(22), pp. 199-215. https://doi.org/10.17163/ret.n22.2021.01 


\section{Introducción}

La digitalización de la economía ha transformado las actividades económicas para que sean más efectivas y flexibles, la crisis económica derivada por el Covid-19 y las medidas sanitarias impuestas por los gobiernos de todo el mundo generan entornos donde las transacciones por medios digitales son más importantes (Yeong et al., 2019). De enero de 2019 a abril de 2021 en plena crisis, el valor total de mercado de las criptomonedas se ha multiplicado 15 veces con un valor de mercado aproximado de un trillón de dólares (anglosajón), equivalente al PIB de 2021 de países como México, España o Suiza, habiendo más de 10000 criptomonedas diferentes en el mercado (Coinmarketcap, 2021), por lo que un estudio sobre el tema puede ayudar a las empresas o gobiernos a conocer las ventajas de las criptomonedas, como es el caso del yuan digital y la evolución de la infraestructura de los mercados financieros ya que existen más de 100 millones de usuarios confirmados en el mundo (Blandin et al., 2020).

Últimamente se han logrado avances significativos respecto a la comprensión de la confianza en el comercio electrónico y, por lo tanto, en las criptomonedas ya que la confianza se vuelve más significativa en estas áreas siendo difícil verificar las regulaciones, usos y costumbres en el comercio y en todas las transacciones digitales, ahora las redes de comunicación están más vinculadas con los servicios financieros ya que la banca móvil ofrece más beneficios respecto a la banca tradicional, como la verificación de saldo y la transferencia de fondos de manera instantánea (Afshan \& Sharif, 2016; Gefen et al., 2003; Kim et al., 2009). El comercio a través de medios electrónicos va más allá de su interfaz, la confianza es un elemento que va más allá de las relaciones transaccionales de los usuarios, especialmente las que contienen algún aspecto de riesgo (Gefen et al., 2003).

Debido a su particular característica de virtualidad, las criptomonedas podrían implicar un gran riesgo e incertidumbre que reduce la confianza para ser adoptadas, la confianza, corresponde a la expectativa de que los demás no se comportarán de manera oportunista, de que la otra parte cumplirá sus obligaciones a pesar de la dependencia y de la vulnerabilidad de la parte en la que confía, haciendo necesaria cierta regulación, toda vez que, cuando se carece de una reglamentación efectiva mínima en los medios digitales los usuarios tienen que confiar en la tecnología que utilizarán (Gefen et al., 2003; Zhou, 2012). La confianza debe de ser el atributo determinante, incluso más allá de los factores económicos (Gefen et al., 2003; Gefen \& Straub, 2004), de ahí la pertinencia de conocer cómo se puede confiar en las transacciones digitales cuando no hay interacciones personales y cómo tener confianza en una criptomoneda cuando no hay instituciones que la respalden, por estas razones es necesario conocer cuáles son los factores en los que se basa la confianza y si determinan el uso de bitcoin. Para ello, se revisa el concepto de confianza en relación con el bitcoin y las criptomonedas, las teorías que la explican y cómo se puede medir; en segundo lugar, se analizan los factores que generan confianza, con el fin de conocer cómo estos factores contribuyen a lograr los objetivos de este trabajo de investigación; en tercer lugar, se presenta un modelo conceptual incorporando su análisis metodológico; en cuarto lugar se presentan los resultados de la aplicación del modelo y finalmente se realiza una discusión de los resultados que incluye las conclusiones. 


\subsection{Marco teórico}

\subsubsection{El bitcoin}

El bitcoin ha surgido como una nueva alternativa a los medios de pago, con múltiples beneficios para los usuarios como anonimidad y bajas tarifas de transacción (Yeong et al., 2019). Consiste en un sistema de pagos público y descentralizado que se basa en la tecnología de Blockchain, la cual consiste en un registro de todas las transacciones que se llevan a cabo (Inoue, 2016). La tecnología de Blockchain exige que todos los procedimientos del sistema de pagos, sean realizados por usuarios voluntarios que aportan capacidad de proceso a través de sus computadoras, ellos confirman las transacciones y procesan los datos que se añaden a la cadena, todas las transacciones y nuevos bloques generan comisiones que son repartidas equitativamente (Sadhya et al., 2018).

\subsubsection{La confianza}

La confianza ha sido planteada como una construcción unidimensional ignorando la gran cantidad de evidencias que sugieren que es una construcción compleja y de una naturaleza multidimensional, por lo que se hace una revisión de los factores que conducen a la confianza, cada uno contribuye a una perspectiva de este concepto ya que puede tomar diferentes formas y relaciones, por ello la confianza en los medios digitales es una combinación de múltiples factores (Gefen et al., 2003; Kim \& Prabhakar, 2004). La confianza una construcción multidimensional, que se da en los medios electrónicos, a pesar de la falta de interacción humana, lo que explica su importancia (Gefen \& Straub, 2004).

La confianza se puede definir como una combinación de confiabilidad, integridad y benevolencia de los proveedores electrónicos con la creencia de que estos generan intenciones de comportamiento entre los consumidores, definición que separa la confianza de las intenciones reales de uso, ya que la confianza afecta positivamente la intención (Gefen et al., 2003; Mayer et al., 1995). En los medios digitales no hay un contrato detallado que otorgue protección legal o que obligue a las partes cuando este es insuficiente, por lo que la experiencia que se produce entre los usuarios genera confianza ya que establece credibilidad por parte del vendedor de que proporcionará lo prometido (Villarroel-Puma \& Berenguer-Contri, 2020; Gefen et al., 2003).

Existen diferentes antecedentes de la confianza que ayudan a entender mejor este concepto por ejemplo: 1) la familiaridad, sugiere que la confianza se desarrolla con el tiempo, que resulta de la experiencia entre las partes (Gefen et al., 2003); 2) confianza basada en calculo: se basa en principios económicos ya que la creación de confianza implica un proceso calculador (Gefen et al., 2003); 3) confianza basada en la institución, se refiere a que la evaluación de que la transacción será exitosa en función de que es habitual en situaciones de este tipo (Gefen et al., 2003); 4) confianza basada en garantías estructurales, se refieren a la evaluación del éxito debido a las redes de seguridad, recursos legales y regulaciones (Gefen et al., 2003); 5) la propensión a confiar, se refiere a que la confianza depende de una disposición del individuo a confiar (Gefen \& Straub, 2004); 6) la predictibilidad: es la creencia de que el vendedor se comportará de manera confiable (Gefen \& Straub, 2004).

Lo anterior demuestra que ha surgido un nuevo tipo de confianza basada en la tecnología, que debe de ser contemplada de manera multidimensional, con la finalidad de conocer que factores la explican mejor, lo que analizaremos con más detalle más adelante. 


\subsubsection{El riesgo percibido}

El riesgo percibido es la disposición de los individuos hacia el riesgo (Diez-Farhat, 2020), la literatura señala que es un elemento muy importante sobre todo en aquellas relaciones impersonales en las que se contempla alguna situación de riesgo y que no debe analizarse la confianza sin contemplar el riesgo sobre todo en relaciones donde existe incertidumbre, la inclusión de esta variable es muy importante ya que la decisión de usar o no una tecnología se basa en el análisis del costo-beneficio por lo que el riesgo debe de jugar un papel central (Gefen et al., 2003; Pavlou, 2003). Existen estudios que han analizado el riesgo encontrando que los usuarios perciben un alto riesgo al usar servicios electrónicos y criptomonedas ya que es un factor que obstaculiza la intención del comportamiento (Alalwan et al., 2018; Esmaeilzadeh et al., 2019; Xie et al., 2017).

En el presente contexto adoptamos esa posición frente al riesgo, como una connotación negativa sobre las intenciones del usuario de utilizar alguna tecnología que lleve a un individuo a incurrir en una perdida en la búsqueda de un resultado.

\subsubsection{Evidencia empirica}

La adopción en las criptomonedas ha sido analizada de diferentes maneras. En la Tabla 1 se hace una breve descripción sobre los estudios de aceptación y confianza que se han hecho sobre las criptomonedas y bitcoin, en ella se observan qué teorías de aceptación se han aplicado, las metodologías que se han utilizado para analizar datos y cuáles han sido los principales resultados. A pesar de la aparente madurez derivada de esta comparación, ninguno de ellos analiza la confianza multidimensional ni incluye el riesgo percibido.

Se encontró que todos los trabajos revisados presentan una adecuada aplicación de las diferentes variables teóricas de los modelos de aceptación, integrándolos con el concepto de confianza, en todos ellos se comprobó exitosamente la confiabilidad y validez de los modelos, en todos los estudios se demostró que la confianza es uno de los elementos más importantes por lo que se debe analizar a profundidad este elemento (López-Zambrano \& Camberos-Castro, 2020; Mahomed, 2017; Roos, 2015; Shahzad et al., 2018). Se demuestra que existen pocos estudios que analizan la confianza en bitcoin o criptomonedas y que de los cuatro arriba mencionados ninguno la valoriza de forma multidimensional, tampoco se analiza el riesgo percibido como un complemento para la confianza. Si bien es cierto, que la confianza es un factor determinante en el uso de las criptomonedas, es necesario hacer un análisis multidimensional e incluir el riesgo percibido para conocer qué elementos son los más importantes (Gefen et al., 2003). 


\section{Tabla 1. Revisión de evidencia empírica}

\begin{tabular}{|l|l|l|l|l|}
\hline \multicolumn{1}{|c|}{ Autor } & $\begin{array}{c}\text { Teoría y } \\
\text { conceptos }\end{array}$ & \multicolumn{1}{|c|}{ Contexto } & Metodología & \multicolumn{1}{|c|}{ Resultados } \\
\hline $\begin{array}{l}\text { Mahomed } \\
(2017)\end{array}$ & $\begin{array}{l}\text { UTAUT2 y } \\
\text { confianza }\end{array}$ & $\begin{array}{l}\text { Adopción de } \\
\text { las criptomo- } \\
\text { nedas }\end{array}$ & $\begin{array}{l}\text { Regresión lineal } \\
\text { múltiple }\end{array}$ & $\begin{array}{l}\text { La confianza el 25\%. El } \\
\text { modelo explica el 29\% de } \\
\text { la intención. }\end{array}$ \\
\hline Roos (2015) & $\begin{array}{l}\text { UTAUT2 y } \\
\text { Confianza }\end{array}$ & $\begin{array}{l}\text { Adopción de } \\
\text { las cripto- } \\
\text { monedas en } \\
\text { PyMES }\end{array}$ & $\begin{array}{l}\text { Análisis descrip- } \\
\text { tivo }\end{array}$ & $\begin{array}{l}\text { El estudio demuestra que la } \\
\text { confianza es el factor más } \\
\text { importante. }\end{array}$ \\
\hline $\begin{array}{l}\text { Shahzad et al. } \\
(2018)\end{array}$ & $\begin{array}{l}\text { TAM, Con- } \\
\text { ciencia y } \\
\text { confianza }\end{array}$ & $\begin{array}{l}\text { Adopción de } \\
\text { las cripto- } \\
\text { monedas en } \\
\text { China }\end{array}$ & SEM & $\begin{array}{l}\text { El modelo logra explicar el } \\
\text { 51 \% de la intención, sien- } \\
\text { do la confianza el factor } \\
\text { determinante. }\end{array}$ \\
\hline $\begin{array}{l}\text { López y } \\
\text { Camberos } \\
(2020)\end{array}$ & $\begin{array}{l}\text { UTAUT2 y } \\
\text { Confianza }\end{array}$ & $\begin{array}{l}\text { Adopción de } \\
\text { bitcoin en } \\
\text { México. }\end{array}$ & PLS-SEM & $\begin{array}{l}\text { El modelo logra explicar el } \\
79.3 \% \text { de la intención de } \\
\text { uso y 30.6 \% del uso real. }\end{array}$ \\
\hline
\end{tabular}

Fuente: López-Zambrano, y Camberos-Castro (2020); Mahomed (2017); Roos (2015); Shahzad et al. (2018)

\subsubsection{Integración del modelo conceptual}

$\mathrm{Al}$ analizar los diferentes modelos y teorías de confianza nos encontramos que el modelo de Gefen (2003) es el que mejor se adapta ya que contempla parte de la confianza inicial considerando que el fenómeno de las criptomonedas es relativamente nuevo, ya que está vigente desde 2008 (Nakamoto, 2008). A su vez, las variables de este modelo contemplan otras dimensiones que logran sintetizar diferentes conceptos en uno solo (Aljaafreh et al., 2014; Kim et al., 2009; Sun et al., 2017).

El riesgo es analizado unidimensionalmente debido a que la escala de riesgo percibido que utilizó Xie et al. (2017) fue validada y demostró ser robusta contemplando todas las dimensiones propuestas por Featherman y Pavlou (2003) y Lee (2009).

Por lo tanto, se propone el modelo planteado en la Figura 1 donde se observan las variables exógenas de confianza basada en el cálculo, confianza basada en garantías estructurales, normalidad situacional y riesgo percibido que afectan a las variables endógenas de confianza, intención de uso y uso real. Se constata que la confianza y el riesgo percibido son los elementos más importantes para que el bitcoin sea aceptado. El objetivo del modelo es tratar de medir las relaciones de un usuario al iniciar el contacto con una nueva tecnología y cómo esta se incrementa con la interacción generando la confianza necesaria, sin ella, el usuario no usará dicha tecnología (Alalwan et al., 2017; Aljaafreh et al., 2014; Sun et al., 2017).

A continuación, para orientar mejor el análisis, se describen diferentes hipótesis de cómo los factores del modelo integrado afectan a la intención de uso:

H1. La confianza basada en cálculo (BC) afecta positivamente la confianza en el bitcoin

La confianza basada en cálculo se da cuando el vendedor no tiene nada que ganar al no ser confiable, pero la tecnología de Blockchain convierte al bitcoin en un medio de pago descentralizado que no es controlado por ninguna persona o institución, que lo convierte en un servicio íntegro y fidedigno, aunque hay empresas e individuos que 
prestan sus servicios a través de bitcoin como billeteras en línea (wallets) o casas de cambio (exchangers) que generan ingresos a través de comisiones, se espera que estas empresas tengan mucho que perder por no ser confiables y mucho que ganar con el uso de Blockchain.

H2. Las percepciones de las garantías estructurales (GE) integradas en el bitcoin afectan positivamente en la confianza de los usuarios

La confianza basada en garantías estructurales se refieren a la evaluación del éxito debido a sus recursos de la tecnología de Blockchain, que respalda al bitcoin y permite tener una red de pagos segura que es imposible de quebrantar (Sadhya et al., 2018). Si bien es cierto que actualmente la mayoría de los países del mundo cuentan con leyes que regulan el uso de bitcoin y a las empresas que ofrecen sus servicios a través de él aunque estas pueden tener aún lagunas. Se espera que las empresas que ofertan sus servicios a través de bitcoin, sobre todo las que operan en la nube, deben ofrecer las garantías estructurales necesarias.

H3. La percepción de normalidad situacional (NS) afecta positivamente la confianza en el bitcoin

La confianza basada en la institución en cuanto a normalidad situacional se refiere a que las transacciones se logran debido a que es lo habitual, a diferencia de la familiaridad la normalidad situacional no trata con el conocimiento de la tecnología, sino que se refiere a la medida en que la interacción con determinada tecnología es normal en comparación con otras tecnologías similares (Gefen et al., 2003). Cuando los usuarios realizan transferencias por medio de bitcoin se espera que estas sean exitosas o cuando estos guardan sus activos en una billetera de bitcoin esperan que estén resguardados, similar al de la banca en línea.

H4. La familiaridad (FL) con el uso de bitcoin aumenta positivamente la confianza que se tiene en él

La familiaridad sugiere que la confianza se desarrolla con el tiempo de interacción con la tecnología que resulta de la experiencia, debe aumentar la confianza ya que implica una mayor experiencia derivada de los conocimientos acumulados (Gefen et al., 2003). El uso de bitcoin implica un involucramiento progresivo por parte del usuario ya que es necesario contar con conocimientos técnicos y financieros para su uso, por lo que un aumento en el conocimiento y las interacciones exitosas previas acumuladas propician niveles más altos de confianza.

H5. La confianza (CZ) afecta positivamente la intención de los usuarios de utilizar bitcoin

La confianza se puede definir como una combinación de confiabilidad, integridad, benevolencia y capacidad del usuario de una determinada tecnología con la creencia de que se pueden generar intenciones de comportamiento, por lo que elevados niveles de confianza como creencias específicas sobre una tecnología aumentan la intención de uso (Alalwan et al., 2017; Gefen et al., 2003). La confianza en el bitcoin ayuda al usuario a descartar subjetivamente situaciones indeseables derivadas de su uso, sobre todo por parte de los prestadores de servicios.

H6. El riesgo percibido (RP) tiene un efecto negativo en la intención de uso del bitcoin

El riesgo percibido se refiere a la creencia de un individuo de incurrir en una pérdida en la búsqueda de un resultado, el interés en este factor se debe a la alta incer- 
tidumbre, la intangibilidad y la ausencia de interacción humana de las transacciones en línea (Alalwan et al., 2018; Xie et al., 2017). Los riesgos financieros a la hora de usar bitcoin han demostrado ser uno de los principales obstáculos al adoptarlo debido a su alta volatilidad, así como las posibles violaciones a la seguridad que puedan sufrir los proveedores de servicios de bitcoin como billeteras o casa de intercambio (Abramova \& Böhme, 2016; Sas \& Khairuddin, 2015), por lo que un aumento en la sensación de riesgo en los usuarios afecta negativamente en la intención de usar bitcoin.

H7. La intención de uso (IU) afecta positivamente el uso real (UR) de bitcoin

La intención de uso supone que el uso real de bitcoin se predice por la disposición de los usuarios a adoptar esta tecnología (López-Zambrano \& Camberos-Castro, 2020).

\section{Figura 1. Modelo conceptual}

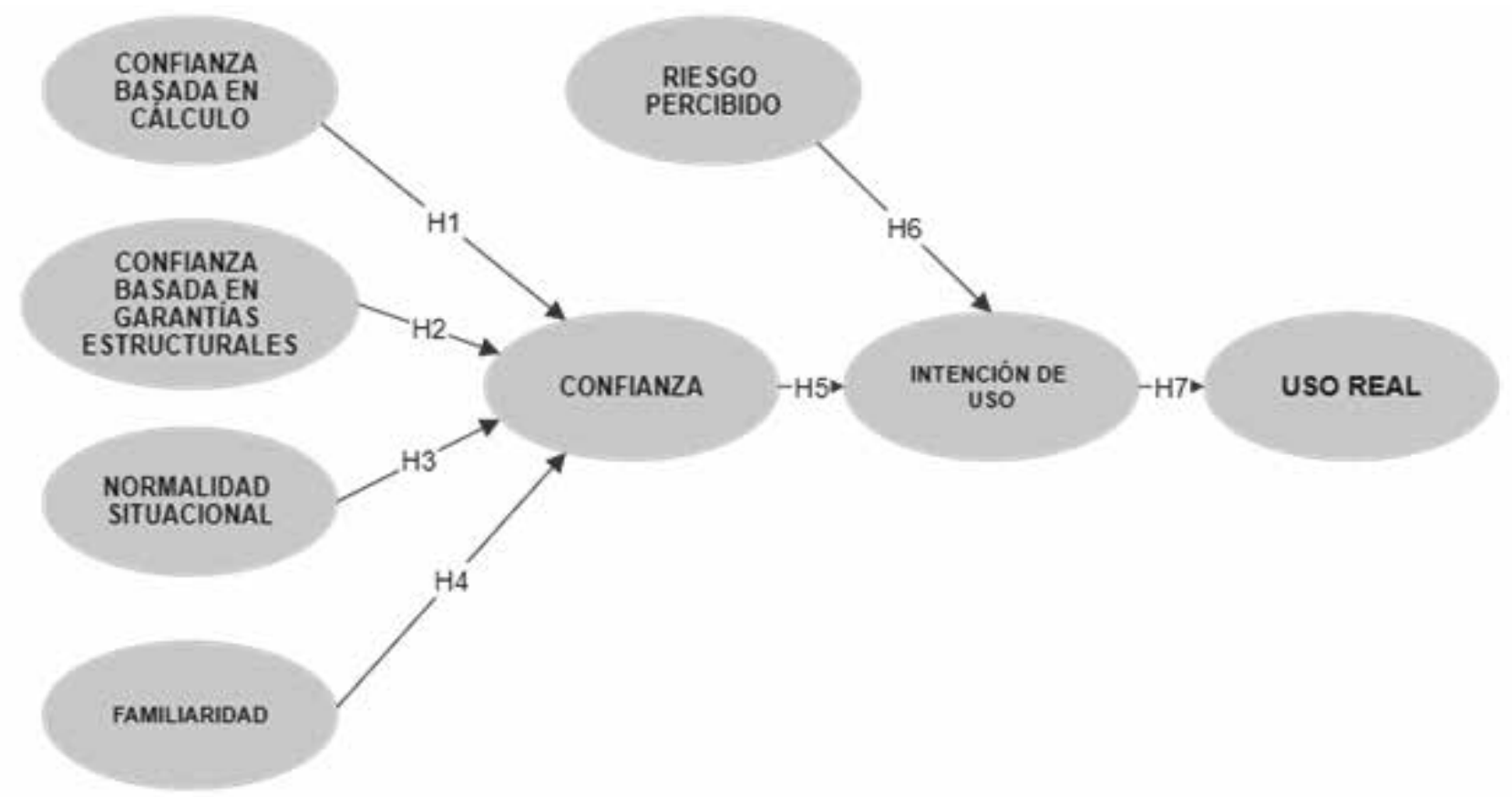

Fuente: Elaboración propia con datos de Gefen (2003).

\section{Metodología}

El modelo de ecuaciones estructurales (SEM) es una técnica para estimar relaciones causales aplicando una combinación de datos estadísticos que permite a los investigadores comprobar teorías, conceptos y verificar las relaciones entre variables en niveles teóricos (Hair et al., 2018; Hair et al., 2012; Oliveira et al., 2016; OwusuKwateng et al., 2019). El modelo SEM puede ser abordado desde la técnica basada en covarianza (CB-SEM) la cual minimiza la discrepancia entre matrices de covarianza o por medio de ecuaciones estructurales por mínimos cuadrados parciales (PLS-SEM) que maximiza la varianza explicada de las variables (Hair et al., 2016; Oliveira et al., 2016). Esta segunda técnica es menos restrictiva en cuanto a la distribución de los datos y señala que el tamaño de la muestra debe de ser mayor a 10 veces el número de trayectorias que apuntan a alguna variable, en este caso el tamaño mínimo de muestra a considerar es de 70 (Hair et al., 2016; Zhou et al., 2010). Se evaluó la distribución de la muestra a través de la prueba Kolmogorov-Smirnov (K-S) detectando que los datos no están distribuidos normalmente ya que los niveles de significancia fueron menores a 
0.05. Basándonos en lo anterior el modelo PLS-SEM es el método más consistente para este tipo de estudios añadiendo que la naturaleza anónima del uso de las criptomonedas no permite obtener muestras muy grandes (López-Zambrano \& Camberos-Castro, 2020; Oliveira et al., 2014).

Otro de los motivos para utilizar la técnica PLS-SEM es que utiliza indicadores reflectivos y que el estudio trata de predecir constructos fundamentales como la confianza y el riesgo que parten de una investigación exploratoria al ser un fenómeno reciente, siendo el paquete de software estadístico SMART-PLS versión 3.3.2 el más apropiado para el análisis de resultados (Leyva et al., 2014).

\subsection{Medición}

Con base en la revisión de antecedentes planteada en el apartado teórico se elaboró y se probó para mejorarlo y se aplicó un cuestionario electrónico a través de la plataforma Google Forms en idioma español, el cual fue validado frente a un panel de expertos en las áreas de economía y finanzas, los ítems y las escalas relacionadas con la confianza fueron adaptados de Gefen (2003), los ítems y la escala de riesgo percibido fueron adaptadas de Xie et al. (2017), para medir los constructos se utilizaron 26 indicadores reflectivos (preguntas) relacionados con cada variable. La unidad de análisis del presente estudio son los usuarios de Bitcoin en México y las respuestas son medidas a través de una escala Likert de 7 puntos en niveles de intervalo que van desde "totalmente en desacuerdo" a "totalmente de acuerdo". La frecuencia de uso se midió a través de una escala que va desde "nunca" a "varias veces al día". Las preguntas demográficas que se incluyen son referentes a la edad, género, y nivel educativo con el propósito de conocer el entorno de los usuarios. Con la finalidad de hacer una prueba piloto se distribuyó el cuestionario a través de la plataforma Airbit Club relacionada con criptomonedas, las evidencias preliminares indicaron que las escalas eran válidas y confiables; cabe señalar que estas respuestas no fueron incluidas en los resultados finales.

\subsection{Datos}

Con la finalidad de detectar el mayor número de usuarios de bitcoin se optó por un muestreo no probabilístico por conveniencia toda vez que la naturaleza anónima de esta criptomoneda dificulta la obtención de datos, una de las ventajas de este enfoque es obtener información confiable y precisa ya que los encuestados están familiarizados con el tema de estudio (Owusu-Kwateng et al., 2019). Por medio del software Autonetworks se lograron detectar 13 grupos de usuarios de la red social Facebook únicamente en México ya que tomaría demasiado tiempo considerar otros países debido a que el software utilizado solo permite enviar 100 mensajes personalizados por día, de los cuales se extrajeron los nombres de 9000 usuarios a los que se les hizo llegar la encuesta de forma masiva por medio de mensajes personalizados logrando obtener 174 respuestas validadas con herramientas estadísticas que se explican a detalle en la siguiente sección. Se tomaron en cuenta solo usuarios de México.

\section{Resultados}

El perfil de respuesta de los encuestados se observa en la Tabla 2 donde solo el $11 \%$ equivale a mujeres, la edad promedio es de 32 años y la mayoría (76\%) tiene grado universitario. 
Tabla 2. Información demográfica

\begin{tabular}{|l|r|r|}
\cline { 2 - 3 } \multicolumn{1}{l|}{} & \# & \multicolumn{1}{c|}{$\%$} \\
\hline Género & & \\
\hline Masculino & 154 & 88.5 \\
\hline Otro & 19 & 10.9 \\
\hline Edad & 1 & 0.6 \\
\hline Menores de 20 & 6 & 3.4 \\
\hline $21-25$ & 43 & 24.7 \\
\hline $26-35$ & 69 & 39.7 \\
\hline $36-45$ & 39 & 22.4 \\
\hline Mayores a 46 & 17 & 9.8 \\
\hline Educación & & \\
\hline Primaria & 2 & 1.1 \\
\hline Secundaria & 6 & 3.4 \\
\hline Preparatoria & 34 & 19.5 \\
\hline Licenciatura & 104 & 59.8 \\
\hline Posgrado & 28 & 16.1 \\
\hline
\end{tabular}

Fuente: Elaboración propia.

\subsection{Análisis del modelo de medición}

Con el propósito de conocer la confiabilidad y validez del modelo, se calcula la consistencia interna, la confiabilidad del indicador, la validez convergente, el promedio de la varianza extraída (AVE) y la validez discriminante (Hair et al., 2014).

Para medir la consistencia interna del modelo de medición se evaluó la confiabilidad compuesta (CC) y el alfa de Cronbach cuyos valores deben de ser mayores a 0.70 como se indica en la Tabla 3 (Alalwan et al., 2017; Oliveira et al., 2014).

Tabla 3. Consistencia interna y validez

\begin{tabular}{|c|c|c|c|c|c|}
\hline Variable & $\begin{array}{c}\alpha \text { de } \\
\text { Cronbach }\end{array}$ & $\mathrm{CC}$ & AVE & Ítem & Indicador \\
\hline Basada en Cálculo & 0.821 & 0.893 & 0.735 & $\begin{array}{l}\mathrm{BC} 1 \\
\mathrm{BC} 2 \\
\mathrm{BC} 3\end{array}$ & $\begin{array}{l}0.861 \\
0.854 \\
0.856\end{array}$ \\
\hline Confianza & 0.903 & 0.933 & 0.777 & $\begin{array}{l}\mathrm{CZ1} \\
\mathrm{CZ2} \\
\mathrm{CZ3} \\
\mathrm{CZ4}\end{array}$ & $\begin{array}{l}0.929 \\
0.824 \\
0.853 \\
0.915\end{array}$ \\
\hline \multirow[t]{2}{*}{ Familiaridad } & \multirow[t]{2}{*}{0.735} & \multirow[t]{2}{*}{0.849} & \multirow[t]{2}{*}{0.66} & FL1 & 0.584 \\
\hline & & & & $\begin{array}{l}\text { FL2 } \\
\text { FL3 }\end{array}$ & $\begin{array}{l}0.908 \\
0.902\end{array}$ \\
\hline
\end{tabular}




\begin{tabular}{|c|c|c|c|c|c|}
\hline Variable & $\begin{array}{c}\alpha \text { de } \\
\text { Cronbach }\end{array}$ & $\mathrm{CC}$ & AVE & Ítem & Indicador \\
\hline \multirow{4}{*}{$\begin{array}{l}\text { Garantías } \\
\text { estructurales }\end{array}$} & \multirow{4}{*}{0.856} & \multirow[t]{4}{*}{0.903} & \multirow[t]{4}{*}{0.7} & GE1 & 0.874 \\
\hline & & & & GE2 & 0.865 \\
\hline & & & & GE3 & 0.892 \\
\hline & & & & GE4 & 0.702 \\
\hline \multirow{4}{*}{ Intención de uso } & \multirow{4}{*}{0.888} & \multirow[t]{4}{*}{0.923} & \multirow{4}{*}{0.75} & IU1 & 0.869 \\
\hline & & & & IU2 & 0.832 \\
\hline & & & & IU3 & 0.936 \\
\hline & & & & IU4 & 0.823 \\
\hline \multirow{3}{*}{$\begin{array}{l}\text { Normalidad } \\
\text { situacional }\end{array}$} & \multirow{3}{*}{0.867} & \multirow{3}{*}{0.918} & \multirow{3}{*}{0.789} & NS1 & 0.876 \\
\hline & & & & NS2 & 0.882 \\
\hline & & & & NS3 & 0.906 \\
\hline \multirow[t]{4}{*}{ Riesgo percibido } & \multirow[t]{4}{*}{0.794} & \multirow[t]{4}{*}{0.863} & \multirow{4}{*}{0.617} & RP1 & 0.894 \\
\hline & & & & RP2 & 0.882 \\
\hline & & & & RP3 & 0.612 \\
\hline & & & & RP4 & 0.717 \\
\hline Frecuencia & 1 & 1 & 1 & FREC & - \\
\hline
\end{tabular}

Fuente: Elaboración propia.

La fiabilidad del indicador nos ayuda a conocer la validez convergente del modelo, al eliminar un indicador nos dice si la confiabilidad compuesta aumenta, los valores deben ser mayores a 0.5 como se observa en la Tabla 3 (Hair et al., 2014). Por último, se observa en la Tabla 3 que los valores de AVE son mayores a 0.5, por lo que cada constructo explica más de la mitad de la varianza, estos datos indican que los constructos son válidos y confiables (Hair et al., 2014; Mensah et al., 2020).

Para satisfacer la validez discriminante de las escalas se evalúan utilizando el método Fornell-Larcker que establece que la raíz cuadrada de AVE debe ser mayor que todas las relaciones entre cada constructo, así cada constructo comparte más varianza con sus indicadores que con cualquier otro. En la Tabla 4 se observa que el valor de cada variable es mayor que el de su correlación más alta (Hair et al., 2014; Oliveira et al., 2016).

Tabla 4. Criterio de Fornell-Larcker

\begin{tabular}{|l|l|l|l|l|l|l|l|l|}
\cline { 2 - 9 } \multicolumn{1}{c|}{} & \multicolumn{1}{|c|}{ BC } & \multicolumn{1}{c|}{ CZ } & \multicolumn{1}{|c|}{ FL } & \multicolumn{1}{c|}{ GE } & \multicolumn{1}{c|}{ IU } & \multicolumn{1}{c|}{ NS } & \multicolumn{1}{c|}{ RP } & UR \\
\hline BC & 0.857 & & & & & & & \\
\hline CZ & 0.305 & 0.881 & & & & & & \\
\hline FL & 0.158 & 0.702 & 0.812 & & & & & \\
\hline GE & 0.402 & 0.817 & 0.568 & 0.837 & & & & \\
\hline IU & 0.285 & 0.777 & 0.652 & 0.717 & 0.866 & & & \\
\hline NS & 0.36 & 0.35 & 0.334 & 0.503 & 0.304 & 0.888 & & \\
\hline RP & -0.127 & -0.251 & -0.167 & -0.243 & -0.228 & -0.086 & 0.785 & \\
\hline UR & 0.083 & 0.281 & 0.38 & 0.224 & 0.407 & 0.074 & -0.124 & 1 \\
\hline
\end{tabular}

Fuente: Elaboración propia. 


\subsection{Análisis del modelo estructural}

El modelo estructural se analiza después de haber confirmado la confiabilidad y validez del modelo, las relaciones de causalidad entre las variables independientes y dependientes se analizan a través del coeficiente de determinación (R2) (Leyva-Cordero \& Olague, 2014). Para medir el sesgo de los resultados se analiza la colinealidad de estos con el factor de inflación de varianza (VIF) que debe de fluctuar entre 0.2 y 5 . En la Tabla 5 se observa que los valores de este análisis se encuentran en el rango permitido, por lo que se puede decir que no existe colinealidad entre las variables (Hair et al., 2014; Venkatesh et al., 2012).

Tabla 5. Estadísticas de Multicolinealidad (VIF)

\begin{tabular}{|l|r|r|r|}
\cline { 2 - 4 } \multicolumn{1}{c|}{} & CZ & IU & UR \\
\hline BC & 1.257 & & \\
\hline CZ & & 1.067 & \\
\hline FL & 1.501 & & \\
\hline GE & 1.927 & & \\
\hline IU & & & 1 \\
\hline NS & 1.405 & & \\
\hline RP & & 1.067 & \\
\hline
\end{tabular}

Fuente: Elaboración propia.

La técnica de remuestreo Bootstrapping se utiliza para conocer la importancia de los niveles de significancia de los coeficientes de ruta debido a que el método PLS-SEM utiliza distribuciones no normales (Hair et al., 2012; Owusu-Kwateng et al., 2019). Esta técnica extrae submuestras de los datos originales y estima modelos para cada submuestra, en este caso utilizaron 5000 estimaciones que se utilizaron para calcular el error estándar y así determinar la importancia de cada parámetro utilizando los valores t (Hair et al., 2014). Para este estudio se consideraron valores no significativos los mayores a un error de probabilidad de $5 \%$. Al respecto en la Tabla 6 se muestra que la confianza basada en calculo afecta de forma no significativa a la confianza, al igual que el riesgo percibido sobre la intención de uso.

Tabla 6. Prueba de significancia

\begin{tabular}{|c|c|c|c|}
\hline Trayectoria & $\begin{array}{c}\text { Valor T } \\
\text { (|O/STDEV|) }\end{array}$ & Valor $\mathbf{P}$ & Significancia \\
\hline $\mathrm{BC}->\mathrm{CZ}$ & 0.584 & 0.559 & No Significativa \\
\hline $\mathrm{CZ}->\mathrm{IU}$ & 14.096 & 0 & Significativa \\
\hline $\mathrm{FL}->\mathrm{CZ}$ & 6.143 & 0 & Significativa \\
\hline $\mathrm{GE}->\mathrm{CZ}$ & 10.946 & 0 & Significativa \\
\hline IU -> UR & 4.607 & 0 & Significativa \\
\hline $\mathrm{NS}->\mathrm{CZ}$ & 2.211 & 0.027 & Significativa \\
\hline $\mathrm{RP}->\mathrm{IU}$ & 0.687 & 0.492 & No Significativa \\
\hline
\end{tabular}

Fuente: Elaboración propia.

El coeficiente de determinación $\mathrm{R}^{2}$ es una medida de la precisión predictiva y corresponde a los efectos combinados de las variables exógenas sobre las endógenas 
donde los valores más cercanos a 1 corresponden a un mayor grado de predicción (Hair et al., 2014; Vinzi et al., 2010). Según Hair et al. (2012) el tamaño de muestra recomendado para obtener valores significativos de $\mathrm{R}^{2}$ mayores a 0.25 debe de ser mayor a 144 , por lo que valores de $0.75,0.50$ y 0.25 son considerados substanciales, moderados y débiles respectivamente. $\mathrm{Al}$ respecto, $\mathrm{R}^{2}$ nos ayuda a interpretar la prueba estadística de las hipótesis, en la Figura 2 se observa que las hipótesis H1, H3 y H6 no son significativas, se confirman las hipótesis $\mathrm{H} 2, \mathrm{H} 4, \mathrm{H} 5$ y $\mathrm{H} 7$ siendo las garantías estructurales el elemento más importante explicando el $65.7 \%$ de la confianza y que solo la confianza logra predecir el $60.5 \%$ de la intención de uso.

Figura 2. Resultados del modelo estructural

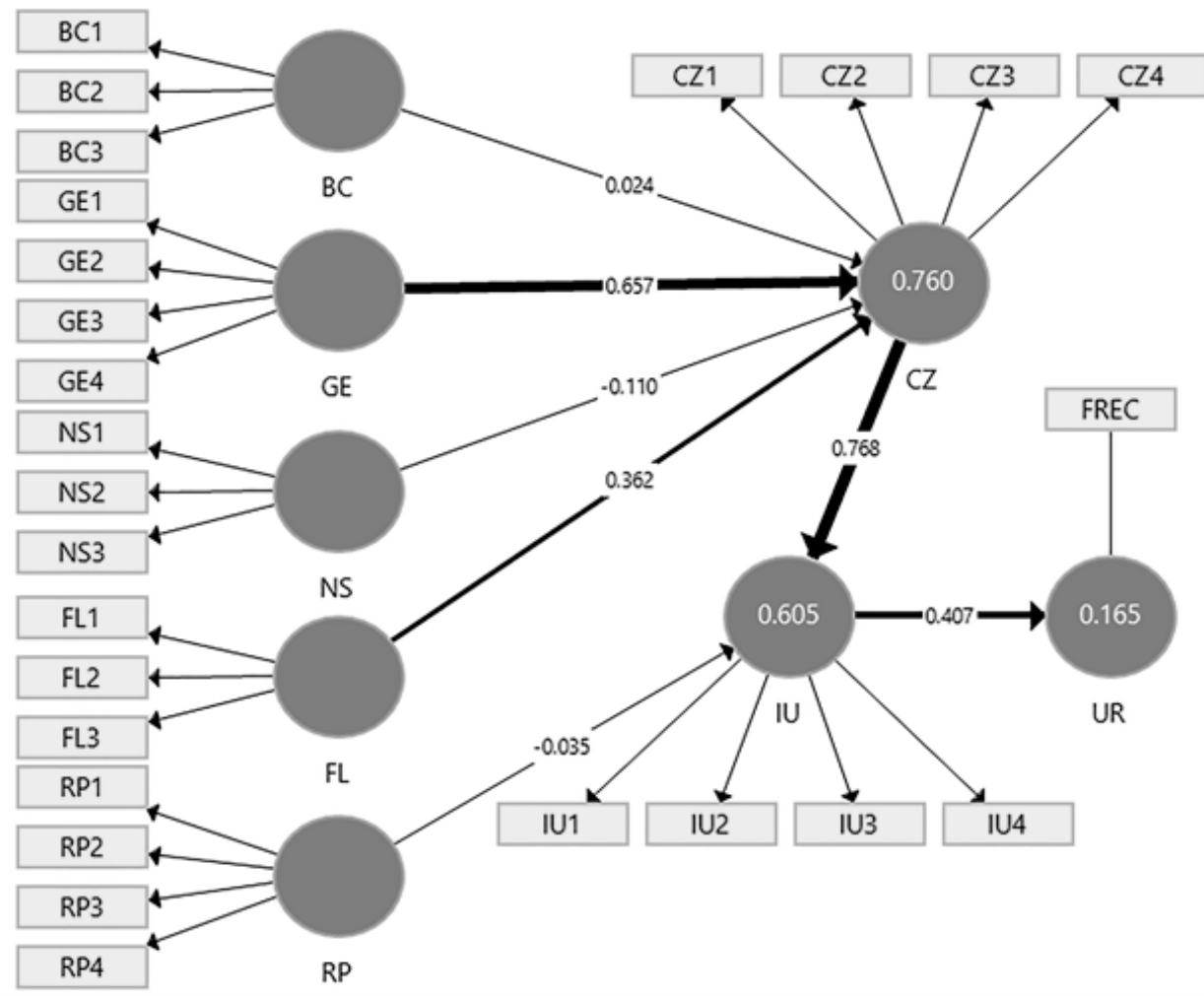

Fuente: Elaboración propia.

Debido a que la estimación de $\mathrm{R}^{2}$ puede no ser suficiente para evaluar todo el modelo estructural, es necesario realizar otros procedimientos (Vinzi et al., 2010), por lo que se utiliza el valor $\mathrm{Q}^{2}$ para conocer la relevancia predictiva del modelo, se mide a través de una técnica de reutilización de datos que omite una parte de estos y predice la parte omitida estimando los parámetros de lo que se obtienen valores que van de $-1 \mathrm{a}+1$, siendo los valores mayores a 0 los que implican una alta relevancia predictiva (Hair et al., 2014). Para considerar los valores de relevancia predictiva como sólidos, estos deben de ser mayores a cero. En la Tabla 7 se observa que todos los valores son mayores y significativos demostrando la capacidad predictiva del modelo teórico (Gu et al., 2019; Min et al., 2018). 
Tabla 7. Relevancia predictiva $\left(Q^{2}\right)$

\begin{tabular}{|l|c|l|}
\hline \multicolumn{1}{|c|}{ Constructo } & $\mathbf{Q}^{2}$ & \multicolumn{1}{c|}{ Significancia } \\
\hline $\mathrm{CZ}$ & 0.581 & Significativa \\
\hline $\mathrm{IU}$ & 0.444 & Significativa \\
\hline $\mathrm{UR}$ & 0.159 & Significativa \\
\hline
\end{tabular}

Fuente: Elaboración propia.

$\mathrm{Al}$ respecto también se evalúa el tamaño de efecto (f2) observando los cambios en $\mathrm{R}^{2}$ cuando un constructo en específico se elimina para evaluar la influencia sobre la variable endógena, siendo valores de $0.02,0.15$ y 0.35 que representan efectos pequeños medianos y grandes respectivamente (Hair et al., 2016). A su vez, también se puede evaluar el tamaño de efecto (f2) de Q2 para conocer el impacto relativo de la relevancia predictiva q2 (Hair et al., 2016). En la Tabla 8 se puede observar que la familiaridad y las garantías estructurales tuvieron un gran efecto sobre la confianza al igual que esta sobre la intención de uso; la intención de uso tuvo un mediano efecto sobre el uso real, en cuanto a las otras trayectorias sus efectos no fueron relevantes. Respecto a q2 solo la FL y GE tuvieron una relevancia predictiva alta sobre la confianza.

Tabla 8. Tamaño de efecto $\left(f^{2}\right)$ e impacto relativo de la relevancia predictiva $\left(q^{2}\right)$

\begin{tabular}{|l|r|r|}
\hline \multicolumn{1}{c|}{ Trayectoria } & \multicolumn{1}{c|}{$\mathbf{f}^{2}$} & \multicolumn{1}{c|}{$\mathbf{q}^{2}$} \\
\hline $\mathrm{BC}->\mathrm{CZ}$ & 0.002 & -0.002 \\
\hline $\mathrm{CZ}->\mathrm{IU}$ & 1.403 & \\
\hline $\mathrm{FL}->\mathrm{CZ}$ & 0.363 & 0.165 \\
\hline $\mathrm{GE}->\mathrm{CZ}$ & 0.933 & 0.418 \\
\hline $\mathrm{IU}->\mathrm{UR}$ & 0.198 & \\
\hline $\mathrm{NS}->\mathrm{CZ}$ & 0.036 & 0.014 \\
\hline $\mathrm{RP}->\mathrm{IU}$ & 0.003 & -0.009 \\
\hline
\end{tabular}

Fuente: Elaboración propia.

\section{Discusión y conclusiones}

Existen diferentes estudios (Gefen, 2000; Gefen et al., 2003; Gefen \& Straub, 2004; Kim et al., 2009; Kim \& Prabhakar, 2004; Sun et al., 2017; Zhou, 2012) que señalan la importancia de analizar la confianza como una variable compleja y multidimensional y no solo como un elemento aislado, hallazgo que fue probado por López y Camberos (2020) en una investigación sobre aceptación de bitcoin en México. En este marco, según las pruebas estadísticas, el modelo de medición de la confiabilidad y validez arrojó resultados positivos (Hair et al., 2012), destacando que tiene altos niveles predictivos al explicar el $60.5 \%$ de la intención de uso, en cuanto a la confianza, el factor que mejor explica es el de las garantías estructurales, con un $65.7 \%$ (Hair et al., 2016).

El análisis de las hipótesis muestra que la confianza basada en cálculos (H1) y la normalidad situacional (H3) son poco significativas ya que su efecto y relevancia son bajos, lo que demuestra que la intención de usar bitcoin no depende de la empresa que facilita el servicio de wallets o exchangers y que los usuarios no consideran aplicaciones similares para utilizar bitcoin (Gefen et al., 2003). La confianza basada en garantías estructurales tiene un efecto significativo, ya que explica la mayor parte de la misma, 
confirmando $\mathrm{H} 2$, siendo el principal elemento de la confianza con un tamaño de efecto (f2) y relevancia predictiva considerados altos; sin embargo, el problema es que la confianza está altamente relacionada con las GE por medio del criterio de Fornell-Larcker, donde las garantías de bitcoin excluyen elementos sospechosos (Gefen et al., 2003), por lo que se recomienda sustituir o combinar este elemento con la confianza para futuros estudios con la finalidad de generar modelos con el menor número de variables posible.

En relación con la familiaridad, se confirma la hipótesis $(\mathrm{H} 4)$ ya que tiene un efecto positivo sobre la confianza al explicar el 36.2\% de esta, además tiene un efecto alto y su relevancia es significativa, por lo que se puede decir que los usuarios de bitcoin consideran necesario revisar y estar actualizados sobre el status de la criptomoneda basados en una interacción con la interfaz de Bitcoin y no a una relación social (Gefen, 2000; Gefen \& Straub, 2004). Respecto al riesgo percibido, los resultados son acordes a la teoría que menciona que debe de tener un impacto negativo sobre la intención de uso, solo que esta es poco significativa por lo que los usuarios de bitcoin no consideren arriesgado su uso (Gefen et al., 2003). El efecto de la confianza sobre la intención de uso es significativo y su relevancia predictiva es alta, demostrando con ello ser un elemento muy importante para explicar la aceptación de bitcoin, por lo que se recomienda integrar los elementos de la confianza con los modelos de aceptación con la finalidad de obtener modelos concisos que explican mejor el surgimiento de las criptomonedas sobre todo a largo plazo (López- Zambrano \& Camberos-Castro, 2020).

Con el creciente impacto que han tenido las criptomonedas y en particular bitcoin sobre la economía, al utilizar una base teórica adecuada en el contexto del usuario integrando teorías, se puede ampliar la aplicabilidad del modelo al hacerlo en nuevas áreas del conocimiento, sobre todo con métodos de análisis estadísticos avanzados (PLSSEM). En este contexto, una de las aportaciones es la inclusión del riesgo percibido, toda vez que demuestra una contribución significativa a la teoría, puesto que se considera uno de los principales obstáculos de la intención de uso. Otra contribución teórica relevante es mostrar la existencia de una moneda descentralizada, cuya confianza no reside en una autoridad monetaria o Banco Central, sino en una tecnología Blockchain, que registra automáticamente el valor de las transacciones de forma precisa, operada por individuos expertos ubicados en granjas mineras y constituye el elemento más importante de las garantías estructurales (GE), que aumenta la confianza en el uso del bitcoin (Gefen et al., 2003).

Para concluir, es importante mencionar que la teoría señala que el riesgo debe analizarse de forma multidimensional para que brinde más elementos, en el caso de las criptomonedas no se recomienda, ya que la connotación negativa es muy poco significativa (Featherman \& Pavlou, 2003; Lee, 2009). Dado que este estudio es de corte trasversal, un estudio longitudinal podría proporcionar un mayor alcance en cuanto a la evolución de los factores en el tiempo, además de recomendar estudios en diferentes regiones y basados en diferentes tipos de criptomonedas con el fin de comparar los resultados.

En la actualidad, las criptomonedas están recibiendo cada vez más atención, principalmente el bitcoin, que en 2021, a pesar de la crisis por la pandemia, ha alcanzado máximos históricos. Tal vez porque el bitcoin existe desde 2008, su aceptación y uso aún no han sido evaluados exhaustivamente por lo que se considera trascendente este tipo de estudios. A ello, hay que añadir que no existen investigaciones que analicen la adopción de las criptomonedas o el bitcoin con pruebas estadísticas de segunda generación. Para llenar esta brecha y cumplir con los objetivos de la presente investigación, se formuló y probó un modelo que integra la confianza multidimensional y el riesgo percibido para medir la intención de uso. Los resultados estadísticos indican la consistencia y validez aunado a un alto poder predictivo al explicar un $60.5 \%$ de la varianza 
de intención de uso y un $16.5 \%$ del uso real de bitcoin. Las garantías estructurales y la familiaridad son los factores más significativos que explican la confianza y por ende la intención de uso de bitcoin, con ello se cumple el objetivo de conocer que estos elementos son clave para explicar la aceptación y uso de bitcoin.

\section{Referencias}

Abramova, S., \& Böhme, R. (2016). Perceived benefit and risk as multidimensional determinants of bitcoin use: a quantitative exploratory study. Proceedings of the Thirty-Seventh International Conference on Information Systems (ICIS 2016), (Zohar 2015), 1-20. https://doi.org/10.1016/j.socscimed.2011.05.023

Afshan, S., \& Sharif, A. (2016). Acceptance of mobile banking framework in Pakistan. Telematics and Informatics, 33(2), 370-387. https://doi.org/10.1016/j.tele.2015.09.005

Alalwan, A. A., Dwivedi, Y. K., \& Rana, N. P. (2017). Factors influencing adoption of mobile banking by Jordanian bank customers: Extending UTAUT2 with trust. International Journal of Information Management, 37(3), 99-110. https://doi.org/10.1016/j.ijinfomgt.2017.01.002

Alalwan, A. A., Dwivedi, Y. K., Rana, N. P., \& Algharabat, R. (2018). Examining factors influencing Jordanian customers' intentions and adoption of internet banking: Extending UTAUT2 with risk. Journal of Retailing and Consumer Services, 40 (August 2017), 125-138. https://doi.org/10.1016/j. jretconser.2017.08.026

Aljaafreh, A., Gill, A. Q., \& Ani, A. Al. (2014). Towards the development of an initial trust model for the adoption of internet banking services in Jordan. Proceedings-Pacific Asia Conference on Information Systems, PACIS 2014, 1-12.

Blandin, A., Pieters, G. C., Wu, Y., Dek, A., Eisermann, T., Njoki, D., \& Taylor, S. (2020). 3rd Global Cryptoasset Benchmarking Study. SSRN Electronic Journal (September). https://doi.org/10.2139/ ssrn.3700822

Coinmarketcap (2021). Cryptocurrency Market Capitalizations | CoinMarketCap. https://coinmarketcap.com/

Diez-Farhat, S. (2020). Factores clave para el desarrollo emprendedor de estudiantes universitarios. Revista Venezolana de Gerencia, (89). https://doi.org/10.37960/revista.v25i89.31386

Esmaeilzadeh, P., Subramanian, H., \& Cousins, K. (2019). Individuals' cryptocurrency adoption: A proposed moderated-mediation model. 25th Americas Conference on Information Systems, AMCIS 2019, (Saito 2015), 1-10.

Featherman, M. S., \& Pavlou, P. A. (2003). Predicting e-services adoption: A perceived risk facets perspective. International Journal of Human Computer Studies, 59(4), 451-474. https://doi. org/10.1016/S1071-5819(03)00111-3

Gefen, D. (2000). E-commerce: The role of familiarity and trust. Omega, 28(6), 725-737. https://doi. org/10.1016/S0305-0483(00)00021-9

Gefen, D., Karahanna, E., \& Straub, D. W. (2003). Trust and TAM in Online Shopping: An Integrated Model. MIS Quarter, 27(1), 51-90. https://doi.org/https://doi.org/10.2307/30036519

Gefen, D., Srinivasan Rao, V., \& Tractinsky, N. (2003). The conceptualization of trust, risk and their electronic commerce: The need for clarifications. Proceedings of the 36th Annual Hawaii International Conference on System Sciences, HICSS 2003, 00(C), 1-10. https://doi.org/10.1109/ HICSS.2003.1174442

Gefen, D., \& Straub, D. W. (2004). Consumer trust in B2C e-Commerce and the importance of social presence: Experiments in e-Products and e-Services. Omega, 32(6), 407-424. https://doi. org/10.1016/j.omega.2004.01.006

Gu, D., Khan, S., Khan, I. U., \& Khan, S. U. (2019). Understanding mobile tourism shopping in Pakistan: An integrating framework of innovation diffusion theory and technology acceptance model. Mobile Information Systems, 2019. https://doi.org/10.1155/2019/1490617

Hair, J. F., Hult, G. T. M., Ringle, C. M., \& Sarstedt, M. (2016). A Primer on Partial Least Squares Structural Equation Modeling (PLS-SEM). En Sage (2nd Editio). SAGE Publications, Ltd. 
Hair, J. F., Black, W. C., Babin, B. J., Anderson, R. E., Black, W. C., \& Anderson, R. E. (2018). Multivariate Data Analysis. https://doi.org/10.1002/9781119409137.ch4

Hair, J. F., Sarstedt, M., Hopkins, L., \& Kuppelwieser, V. G. (2014). Partial least squares structural equation modeling (PLS-SEM): An emerging tool in business research. European Business Review, 26(2), 106-121. https://doi.org/10.1108/EBR-10-2013-0128

Hair, J. F., Sarstedt, M., Ringle, C. M., \& Mena, J. A. (2012). An assessment of the use of partial least squares structural equation modeling in marketing research. Journal of the Academy of Marketing Science, 40(3), 414-433. https://doi.org/10.1007/s11747-011-0261-6

Inoue, B. (2016). Performance of Bitcoin Protocol Variants (Nara Institute of Science and Technology). Nara Institute of Science and Technology. https://bit.ly/3iqxI1b

Kim, G., Shin, B., \& Lee, H. G. (2009). Understanding dynamics between initial trust and usage intentions of mobile banking. Information Systems Journal, 19(3), 283-311. https://doi.org/10.1111/ j.1365-2575.2007.00269.x

Kim, K. K., \& Prabhakar, B. (2004). Initial Trust and the Adoption of B2C e-Commerce: The Case of Internet Banking. Data Base for Advances in Information Systems, 35(2), 50-64. https://doi. org/10.1145/1007965.1007970

Lee, M. C. (2009). Factors influencing the adoption of internet banking: An integration of TAM and TPB with perceived risk and perceived benefit. Electronic Commerce Research and Applications, 8(3), 130-141. https://doi.org/10.1016/j.elerap.2008.11.006

Leyva-Cordero, O., \& Olague, J. T. (2014). Modelo de Ecuaciones Estructurales por el método de mínimos cuadrados parciales (PLS). En Métodos y técnicas cualitativas y cuantitativas aplicables a la investigación en ciencias sociales (pp. 479-497). Ciudad de México.

López-Zambrano, C. R., \& Camberos-Castro, M. (2020). Aceptación y confianza de Bitcoin en México: una investigación empírica. Entre ciencia e ingeniería, 14(28), 16-25. https://doi. org/10.31908/19098367.2011

Mahomed, N. (2017). Understanding consumer adoption of cryptocurrencies. https://bit.ly/3lwRVo1

Mayer, R. C., Davis, J. H., \& Schoorman, F. D. (1995). An integrative model of organizational trust. The Academy of Management Review, 20(3), 709-734. https://doi.org/https://doi.org/10.2307/258792

Mensah, I. K., Chuanyong, L., \& Zeng, G. (2020). Factors determining the continued intention to use mobile money transfer services (MMTS) among university students in Ghana. International Journal of Mobile Human Computer Interaction, 12(1), 1-21. https://doi.org/10.4018/IJMHCI.2020010101

Min, S., So, K. K. F., \& Jeong, M. (2018). Consumer adoption of the Uber mobile application: Insights from diffusion of innovation theory and technology acceptance model. Journal of Travel and Tourism Marketing, 00(00), 1-14. https://doi.org/10.1080/10548408.2018.1507866

Nakamoto, S. (2008). Bitcoin: A peer-to-peer electronic cash system. https://bit.ly/3lGaupX

Oliveira, T., Faria, M., Thomas, M. A., \& Popovič, A. (2014). Extending the understanding of mobile banking adoption: When UTAUT meets TTF and ITM. International Journal of Information Management, 34(5), 689--703. https://doi.org/10.1016/j.ijinfomgt.2014.06.004

Oliveira, T., Thomas, M., Baptista, G., \& Campos, F. (2016). Mobile payment: Understanding the determinants of customer adoption and intention to recommend the technology. Computers in Human Behavior, 61(2016), 404-414. https://doi.org/10.1016/j.chb.2016.03.030

Owusu-Kwateng, K., Osei-Atiemo, K. A., \& Appiah, C. (2019). Acceptance and use of mobile banking: an application of UTAUT2. Journal of Enterprise Information Management, 32(1), 118-151. https://doi.org/10.1108/JEIM-03-2018-0055

Pavlou, P. A. (2003). Consumer acceptance of electronic commerce: Integrating trust and risk with the technology acceptance model. International Journal of Electronic Commerce, 7(3), 101-134. https://doi.org/10.1080/10864415.2003.11044275

Roos, C. (2015). The motivation and factors driving crypto-currency adoption in SMEs. University of Pretoria. 
Sadhya, V., Sadhya, H., Hirschheim, R., \& Watson, E. (2018). Exploring technology trust in Bitcoin: The blockchain exemplar. 26th European Conference on Information Systems: Beyond Digitization - Facets of Socio-Technical Change, ECIS 2018, 1-16. Portsmouth: Association for Information Systems.

Sas, C., \& Khairuddin, I. E. (2015). Exploring Trust in Bitcoin Technology. (December), 338-342. https:// doi.org/10.1145/2838739.2838821

Shahzad, F., Xiu, G. Y., Wang, J., \& Shahbaz, M. (2018). An empirical investigation on the adoption of cryptocurrencies among the people of mainland China. Technology in Society, 55(May), 33-40. https://doi.org/10.1016/j.techsoc.2018.05.006

Sun, B., Sun, C., Liu, C., \& Gui, C. (2017). Research on Initial Trust Model of Mobile Banking Users. Journal of Risk Analysis and Crisis Response, 7(1), 13. https://doi.org/10.2991/jrarc.2017.7.1.2

Venkatesh, V., Thong, J. Y. L., \& Xu, X. (2012). Consumer acceptance and use of information technology: extending the unified theory of acceptance and use of technology. MIS Quarterly, 36(1), 157-178. https://doi.org/https://doi.org/10.2307/41410412

Villarroel-Puma, M. F., \& Berenguer-Contri, G. (2020). eWOM, confianza y engagement: incidencia en el capital de marca. Revista Venezolana de Gerencia, (3). https://doi.org/10.37960/rvg. v25i3.33368

Vinzi, V. E., Chin, W. W., Henseler, J., \& Wang, H. (2010). Handbook of partial least squares concepts. En Handbook of Statistical Bioinformatics. https://doi.org/10.1007/978-3-642-16345-6

Xie, Q., Song, W., Peng, X., \& Shabbir, M. (2017). Predictors for e-government adoption: Integrating TAM, TPB, trust and perceived risk. Electronic Library, 35(1), 2-20. https://doi.org/10.1108/ EL-08-2015-0141

Yeong, Y. C., Kalid, K. S., \& Sugathan, S. K. (2019). Cryptocurrency acceptance: A case of Malaysia. International Journal of Engineering and Advanced Technology, 8(5), 28-38. https://doi.org/10.35940/ ijeat.E1004.0585C19

Zhou, T. (2012). Examining mobile banking user adoption from the perspectives of trust and flow experience. Information Technology and Management, 13(1), 27-37. https://doi.org/10.1007/s10799011-0111-8

Zhou, T., Lu, Y., \& Wang, B. (2010). Integrating TTF and UTAUT to explain mobile banking user adoption. Computers in Human Behavior, 26(4), 760-767. https://doi.org/10.1016/j.chb.2010.01.013 